\title{
Drell-Yan processes at the LHC
}

\author{
Alessandro Vicini* \\ Dipartimento di Fisica, Università degli Studi di Milano and INFN, Sezione di Milano \\ E-mail: alessandro.vicini@mi.infn.it

\section{Giovanni Balossini and Guido Montagna} \\ Dipartimento di Fisica Nucleare e Teorica, Università di Pavia and INFN, Sezione di Pavia \\ E-mail: giovanni.balossini@pv.infn.it, guido.montagna@pv.infn.it
}

\section{Carlo Michel Carloni Calame}

INFN, Italy and School of Physics and Astronomy, Southampton University

E-mail: carlo.carloni.calame@pv.infn.it

\section{Oreste Nicrosini and Fulvio Piccinini}

INFN, Sezione di Pavia

E-mail: oreste.nicrosiniepv.infn.it, fulvio.piccinini@pv.infn.it

\section{Mauro Moretti and Michele Treccani}

Dipartimento di Fisica, Università di Ferrara and INFN, Sezione di Ferrara

E-mail: mauro.moretti@fe.infn.it, michele.treccani@fe.infn.it

We review the status of the calculations of the cross sections of the Drell-Yan processes. We present some results obtained with the new version of the event generator HORACE and some preliminary results of a study aiming at combining consistently EW and QCD corrections to this process.

8th International Symposium on Radiative Corrections (RADCOR)

October 1-5 2007

Florence, Italy

\footnotetext{
${ }^{*}$ Speaker.
} 


\section{EW and QCD tools}

The Drell-Yan process has played a key role in the development of our understanding of QCD and electroweak (EW) interactions in hadron collider experiments, both from the experimental and theoretical point of view [1, 2]. It has become a reference process useful to perform precision measurements of gauge boson masses and widths, to constrain the proton $p d f \mathrm{~s}$ and to monitor the collider luminosity. It represents an important background in the searches of new heavy gauge bosons. Gauge boson production in association with jets is an important background e.g. to the Higgs search in association with vector boson or to Higgs production in vector boson fusion.

Concerning QCD calculations and tools for EW gauge boson production at hadron colliders, the present situation reveals a quite rich structure, that includes next-to-leading-order (NLO) and next-to-next-to-leading-order (NNLO) corrections to $W / Z$ total production rate [3, 4], NLO calculations for $W, Z+1,2$ jets signatures [5, 6] (available in the codes DYRAD and MCFM), resummation of leading and next-to-leading logarithms due to soft gluon radiation [7, 8] (implemented in the Monte Carlo ResBos), NLO corrections merged with QCD Parton Shower (PS) evolution [9] (in the event generator MC@NLO), NNLO corrections to $W / Z$ production in fully differential form [10] (available in the Monte Carlo program FEWZ), as well as leading-order multi-parton matrix elements generators matched with vetoed PS, such as, for instance, ALPGEN [11], MADEVENT [12] and SHERPA [13].

As far as complete $\mathscr{O}(\alpha)$ EW corrections to Drell-Yan processes are concerned, they have been computed independently by various authors in $[14,15,16,17,18]$ for $W$ production and in $[20,21,22]$ for $Z$ production. EW tools implementing exact NLO corrections to $W$ production are DK [14], WGRAD2 [15], SANC [17] and HORACE [18], while ZGRAD2 [20], HORACE [19] and SANC [22] include the full set of $\mathscr{O}(\alpha)$ EW corrections to $Z$ production. The predictions of a subset of such calculations have been recently compared, at the level of same input parameters and cuts, in the proceedings of the Les Houches [23] and TEV4LHC [24] workshops for $W$ production, finding a very satisfactory agreement between the various, independent calculations. A similar comparison is going on also for the neutral current channel in the framework of the Les Houches 2007 workshop "Physics at TeV colliders".

\section{HORACE 3.1}

The event generator HORACE [19], in its present version 3.1, allows the simulation of events of the charged current [18] and neutral current [21] Drell-Yan process. It includes the exact $\mathscr{O}(\alpha)$ EW corrections, matched with a QED Parton-Shower which describes QED multiple photon emission in leading log approximation. When used in combination with the proton $p d f$ set MRS T 2004 QED, it takes into account also photon-induced partonic subprocesses which contribute at $\mathscr{O}(\alpha)$ (charged current) or already at tree level (neutral current). It is a true, fully exclusive event generator, compliant to the Les Houches accord for the format of the saved events.

The matching of the exact $\mathscr{O}(\alpha)$ calculation with the QED Parton-Shower has been implemented according to the formula

$$
d \sigma^{\infty}=F_{S V} \Pi\left(Q^{2}, \varepsilon\right) \sum_{n=0}^{\infty} \frac{1}{n !}\left(\prod_{i=0}^{n} F_{H, i}\right)\left|\mathscr{M}_{n, L L}\right|^{2} d \Phi_{n}
$$



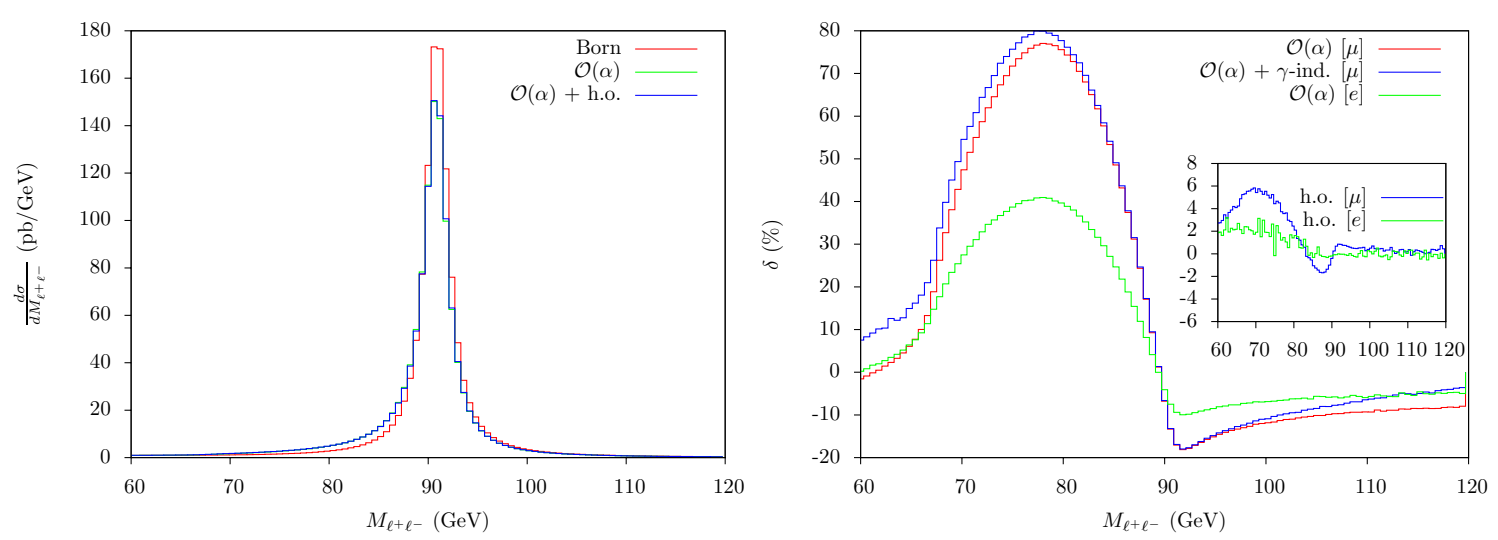

Figure 1: Peak at the $Z$ resonance of the invariant mass distribution in neutral current Drell-Yan at LHC in different approximations (left panel) and relative effect of the radiative corrections (right panel).
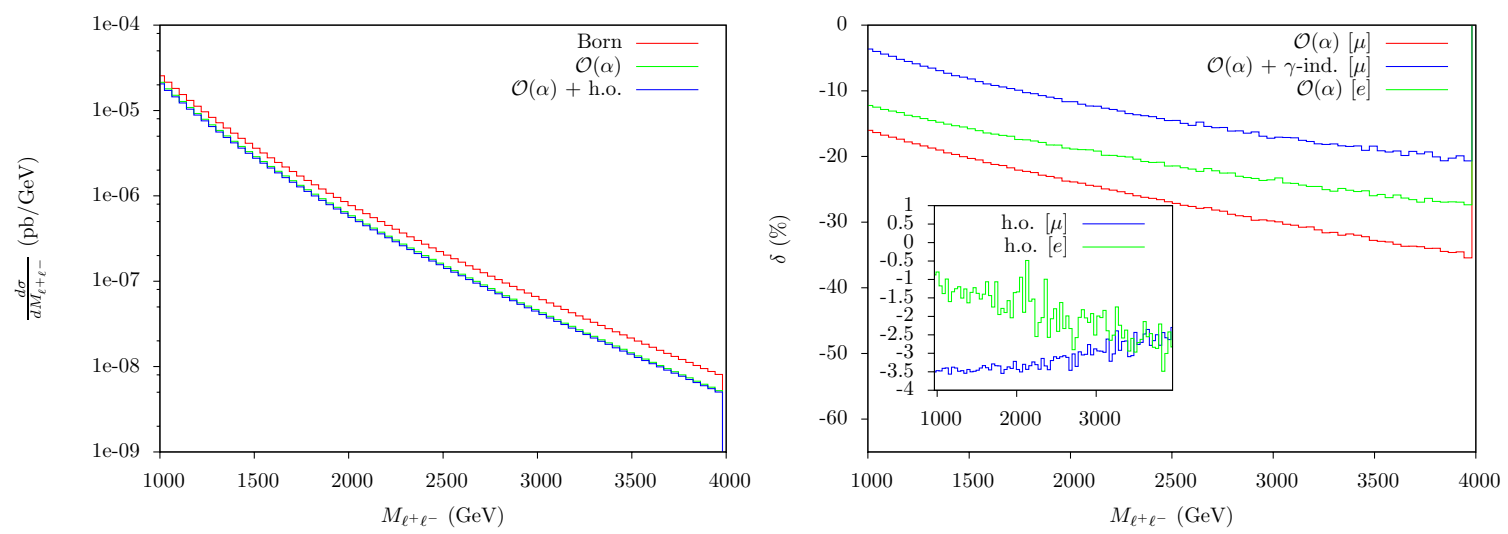

Figure 2: Large mass tail of the invariant mass distribution in neutral current Drell-Yan at LHC in different approximations (left panel) and relative effect of the radiative corrections (right panel).

which can be understood in the following way: 1$)$ the tree-level cross-section ( $n=0$ in the sum) is corrected, in leading-log approximation, to all orders by the Sudakov form factor $\Pi\left(Q^{2}, \varepsilon\right)$, which accounts for virtual and soft-photon emission up to a scale $\varepsilon$ in a hard-scattering process characterized by a virtuality scale $Q ; 2$ ) the resulting expression is dressed by the QED Parton Shower, with the real bremsstrahlung $n$-photons squared matrix elements in leading-log approximation; 3 ) the correction factors $F_{S V}$ and $F_{H, i}$ provide the remaining $\mathscr{O}(\alpha)$ corrections missing in the leadinglog approximation: in particular, $F_{S V}$ contains the remainder of the soft plus virtual corrections, whereas $F_{H, i}$ gives, for the real photon emission, the correction due to the exact bremsstrahlung matrix element with respect to the Parton Shower approximation.

The effect of the EW corrections on the determination of the $W$ and $Z$ masses is large and is dominated by final-state QED radiation, enhanced by large collinear logarithms. NLO EW corrections induce a shift in the extracted value of $m_{W}$ of the order of $100 \mathrm{MeV}$ and higher-order effects contribute with a further shift of $\sim 10 \%$ of the NLO contribution with opposite sign [25]; the latter can not be neglected, in view of the present accuracy reached at the Tevatron[26] and foreseen at the LHC $\left(\Delta m_{W} \sim 15 \mathrm{MeV}\right)$. In the $Z$ case, $\mathscr{O}(\alpha)$ corrections induce a shift in the extracted value of $m_{Z}$ of the order of $400 \mathrm{MeV}$; similarly to the charged current process, multiple photon emission 

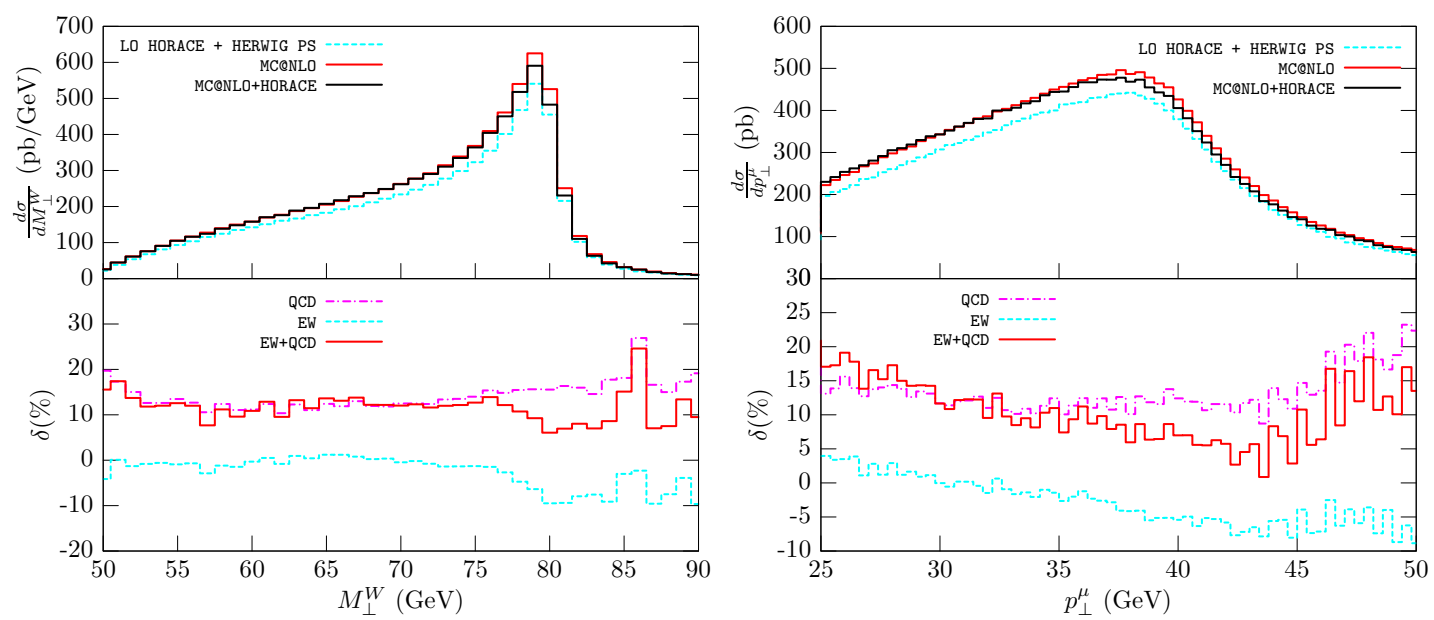

Figure 3: Transverse mass and and transverse lepton momentum tails around the $W$ resonance, in the charged current Drell-Yan process, at the LHC. The effect of the NLO-QCD and of the NLO-EW corrections is displayed.
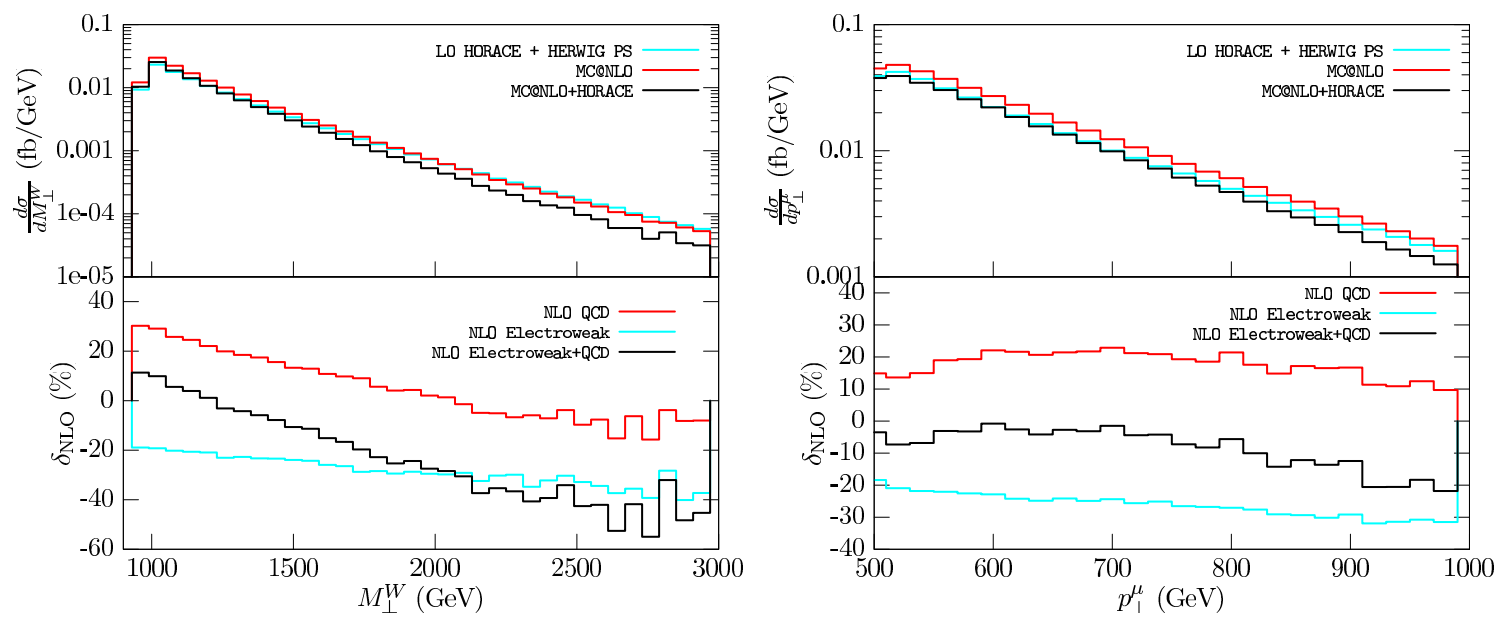

Figure 4: Large transverse mass and and transverse lepton momentum tails, in the charged current Drell-Yan process, at the LHC. The effect of the NLO-QCD and of the NLO-EW corrections is displayed.

yields a further shift of $\sim 10 \%$ of the NLO contribution with opposite sign [27]. An accurate determination of the $Z$ mass allows to calibrate the detectors and affects the systematic error on the $W$ mass. In figure 1 we show the effects at the $Z$ resonance of the exact $\mathscr{O}(\alpha)$ corrections, of the multiple photon emission and of the additional contribution due to the photon-induced subprocesses. It is important to remark that multiple photon emission contributes at the few percent level, in units of the Born cross-section. The tails at large values of the lepton transverse momentum and of the invariant (transverse in the $W$ case) mass distributions receive large negative corrections by the $\mathscr{O}(\alpha)$ virtual corrections and in particular by the so called EW Sudakov logarithms. In figure 2 the invariant mass distribution is shown in different approximations and we can observe that also in this region QED higher orders modify the cross section at the few percent level; furthermore the photon-induced processes give a sizeable $\mathscr{O}(10 \%)$ positive contribution. 


\section{Combining QCD and EW corrections in the charged current case}

In order to obtain a realistic and accurate description of the different observables, it is very important to take into account both EW and QCD corrections. A first attempt in this direction has been presented in [28]. The strategy used in the study presented in this section [29] is the following: 1) different EW and QCD programs have been tuned in order to obtain the same results when computing the same observable with the same set of input parameters at the same order in perturbation theory; 2) the QCD results have been studied using different codes (MC@NLO, ResBos, ALPGEN ) which include higher-order QCD corrections according to different recipes; 3) the effect of the EW corrections has been studied using HORACE; 4) in order to approximately include mixed QCD-EW corrections $\mathscr{O}\left(\alpha \alpha_{s}\right)$ in a factorized form, the events generated by HORACE have been given as input to HERWIG to be convoluted with its initial state QCD Parton-Shower.

The combination of QCD and EW corrections can be cast in the following form [29]:

$$
\left[\frac{d \sigma}{d \mathscr{O}}\right]_{Q C D \oplus E W}=\left\{\frac{d \sigma}{d \mathscr{O}}\right\}_{Q C D}+\left\{\left[\frac{d \sigma}{d \mathscr{O}}\right]_{E W}-\left[\frac{d \sigma}{d \mathscr{O}}\right]_{B o r n}\right\}_{Q C D-P S}
$$

In figures 3 and 4 the results have been obtained in three different approximations: i) a LO calculation convoluted with a QCD Parton Shower, ii) a NLO-QCD calculation matched with a QCD Parton Shower, iii) the combination of NLO-EW and NLO-QCD convoluted/matched with a QCD Parton Shower according to eq.(3.1). We stress the role played by the QCD Parton Shower in providing a correct lowest order description of the shape and of the normalization of the distributions. The relative effect of the various radiative corrections, in the lowest panels of figures 3 and 4, is expressed in units of the Born cross section convoluted with the QCD Parton Shower. Around the $W$ resonance we observe that the QCD Parton Shower broadens the sharply peaked effect of the fixed-order NLO-EW and NLO-QCD corrections. We remark that EW and QCD corrections have, at the peak and in the large transverse mass tail (up to $2 \mathrm{TeV}$ ), opposite signs and tend to compensate each other.

In conclusion the experimental accuracy of the Drell-Yan processes requires precise theoretical predictions, possibly implemented in event generators in order to perform realistic studies of the Tevatron and of the LHC phenomenology. The combination of EW and QCD corrections is necessary to describe properly several important observables.

A.V. wants to thank the organizers for the invitation and for the very pleasant and stimulating atmosphere of the workshop.

\section{References}

[1] P.M. Nadolsky, AIP Conf. Proc. 753 (2005) 158

[2] U. Baur, Electroweak physics at the Tevatron and LHC: Theoretical status and perspectives, arXiv:hep-ph/0511064

[3] G. Altarelli, R. K. Ellis and G. Martinelli, Nucl. Phys. B157 (1979) 461

[4] R. Hamberg, W. L. van Neerven and T. Matsuura, Nucl. Phys. B359 (1991) 343 [Erratum Nucl. Phys. B644 (2002) 403]

[5] W.T. Giele, E.W.N. Glover and D.A. Kosower, Nucl. Phys. B403 (1993) 633 
[6] J.M. Campbell and R.K. Ellis, Phys. Rev. D65 (2002) 113007

[7] C. Balazs and C. P. Yuan, Phys. Rev. D56 (1997) 5558

[8] F. Landry, R. Brock, P.M. Nadolsky and C.-P. Yuan, Phys. Rev. D67 (2003) 073016

[9] S. Frixione and B. R. Webber, JHEP 0206 (2002) 029

[10] C. Anastasiou, L. J. Dixon, K. Melnikov and F. Petriello, Phys. Rev. Lett. 91 (200 3) 182002

C. Anastasiou, L. J. Dixon, K. Melnikov and F. Petriello, Phys. Rev. D69 (2004) 094008

K. Melnikov and F. Petriello, Phys. Rev. Lett. 96 (2006) 231803

K. Melnikov and F. Petriello, Phys. Rev. D74 (2006) 114017

[11] M.L. Mangano, M. Moretti, F. Piccinini, R. Pittau and A.D. Polosa, JHEP 0307 (2003) 001

[12] T. Stelzer and W.F. Long, Comp. Phys. Commun. 81 (1994) 357; F. Maltoni and T. Stelzer, JHEP 02 (2003) 027

[13] T. Gleisberg, S. Höche, F. Krauss, A. Schälicke, S. Schumann and J. Winter, JHEP 0402 (2004) 056

[14] S. Dittmaier and M. Krämer, Phys. Rev. D65 (2002) 0703007

[15] U. Baur and D. Wackeroth, Phys. Rev. D70 (2004) 073015

[16] V. A. Zykunov, Eur. Phys. J. Direct C3 (2001) 9; Phys. Atom. Nucl. 69 (2006) 1522

[17] A. Arbuzov, D. Bardin, S. Bondarenko, P. Christova, L. Kalinovskaya, G. Nanava and R. Sa dykov, Eur. Phys. J. C46 (2006) 407

[18] C. M. Carloni Calame, G. Montagna, O. Nicrosini and A. Vicini, JHEP 0612 (2006) 016 [arXiv:hep-ph/0609170].

[19] http://www.pv.infn.it/ hepcomplex/horace.html

[20] U. Baur, O. Brein, W. Hollik, C. Schappacher and D. Wackeroth, Phys. Rev. D65 (2002) 033007

[21] C. M. Carloni Calame, G. Montagna, O. Nicrosini and A. Vicini, JHEP 0710 (2007) 109 [arXiv:0710.1722 [hep-ph]].

[22] A. Arbuzov, D. Bardin, S. Bondarenko, P. Christova, L. Kalinovskaya, G. Nanava and R. Sadykov, arXiv:0711.0625 [hep-ph].

[23] C. Buttar et al., arXiv:hep-ph/0604120

[24] C. E. Gerber et al. [The TeV4LHC-Top and Electroweak Working Group], arXiv:0705.3251 [hep-ph].

[25] C.M. Carloni Calame, G. Montagna, O. Nicrosini and M. Treccani, Phys. Rev. D69 (2004) 037301

[26] S. Malik, Prepared for 15th International Workshop on Deep-Inelastic Scattering and Related Subjects (DIS2007), Munich, Germany, 16-20 Apr 2007

[27] C. M. Carloni Calame, G. Montagna, O. Nicrosini and M. Treccani, JHEP 0505 (2005) 019 [arXiv:hep-ph/0502218].

[28] Q.-H. Cao and C.-P. Yuan, Phys. Rev. Lett. 93 (2004) 042001; arXiv:hep-ph/0401171

[29] G. Balossini, C.M. Carloni Calame, G. Montagna, M. Moretti, O. Nicrosini, F. Piccinini, M. Treccani and A. Vicini, in preparation and also

AIP Conf. Proc. 870 (2006) 436, Acta Phys. Polon. B 38 (2007) 2347, Contribution prepared for 15th International Workshop on Deep-Inelastic Scattering and Related Subjects (DIS2007), Munich,

Germany, 16-20 Apr 2007 